\title{
9/11 UND diE Folgen IN DER POPMUSIK III. EINE CHRONOLOGISCHE AUSWAHLBIBLIOGRAPHIE
}

\author{
Thomas Phleps
}

Die folgende Bibliographie enthält mit wenigen Ausnahmen Artikel von Journalisten. Äußerungen zum Thema aus der Wissenschaft sind rar, aus der Musikwissenschaft bislang nicht existent. Interessant auch, dass eine große Zahl der Artikel nicht in gedruckter Form, sondern nur in Internetzeitschriften erschien - entsprechend flüchtig sind die Angaben. Allerdings konnte noch Ende Juli 2004 auf fast alle Artikel zugegriffen werden (daher wird allein bei den im Internet nicht weiterhin zugänglichen der letzte Stand angemerkt). Es war mir bei der Auswahl der Artikel wichtig, einen Eindruck vom zeitlichen Verlauf und der Thematik der Diskussion in der Fach-Presse zu vermitteln. Aufgeführt sind relevante Artikel bis April 2003.

\section{Popmusik allgemein}

Corey Moss (2001). "Live, U2, Enya, Aerosmith Songs Become Radio Memorials: News Coverage, Speeches, First-person Testimony Enhance Tracks Reflecting Terrorist Attacks. In: VH1.com, 14. September (http://www.vh1.com/artists/ news/1448887/09142001/aerosmith.jhtml).

Chad Bowar (2001). »Attack on America: The Music World Responds. «In: Suite101, 17. September (http://www.suite101.com/article.cfm/pop_music/80274).

Chet Flippo (2001). »Radio Wants Patriotic Songs - Here They Are. In: MTV News, 20. September (http://www.mtv.com/news/articles/1449014/20010920/story. jhtml).

Michael Billington (2001). »The Culture of Conflict. The Guardian, 29. September (http://www.guardian.co.uk/saturday_review/story/0,3605,559764,00.html).

Jason W. Ocker (2001). »Music and 9/11.« In: TruthinMUSIC, 1. Oktober (http:// www.truthinstuff.com/Music/music911.html; Stand v. 2.9.2003).

Jon Pareles (2001). "Listeners Ask Radio Stations for Songs that Sustain a Weary Soul.« In: The New York Times, 1. Oktober (http://www.whitney-fan.com/nr/ misc/2001/079.shtml).

Randy Lewis (2002). »Eloquent Songs Etched with Post-Attack Sentiments. «In: LoS Angeles Times, 19. Januar (http://events.calendarlive.com/top/1,1419, LLATimes-Music-X!ArticleDetail-50277,00.html; Stand v. 2.9.2003). 
Corey Moss (2002). »Post-9/11 Tribute Albums and Singles: Big Plans, not so Big Results.« In: MTV News, 11. Februar (http://www.mtv.com/news/articles/ 1452229/20020211/story.jhtml).

Walkin' T:-)M (2002). »World's Out Of Tune (1): 'Ground Zero in Song and Poetry.« In: Folk World, the online music magazine, Nr. 21 (März) (http://www. folkworld.de/21/e/ground0.html).

Jeff Chang (2002). »Is Protest Music Dead?« In: Counterpunch, 2. April (http:// www.counterpunch.org/changprotestmusic.html).

Forman, Murray (2002). "Soundtrack to a Crisis: Music, Context, Discourse.«In: Television \& New Media 3, Nr.2 (Mai), S.191-204.

Zalot, Michael C. (2002). "Turning Away from the Television Tape Loop: Characterizing some Local Rock, Pop, \& Country Music Stations' Responses to the Sept. 11, 2001, Terrorist Attacks. « In: The New Jersey Journal of Communication 10, Nr. 1 (Spring), S. 26-48.

Walkin' T:-)M (2002). »World's Out Of Tune (2): >Enduring Freedom< in Song and Poetry.« In: Folk World, the online music magazine, Nr. 22 (Juni) (http://www. folkworld.de/22/e/ground0.html).

Bill Friskics-Warren (2002). »Voices of Reason.«In: PopCULT, 24. Juli (http://www. polarity1.com/pcrr34.html).

Kris Axtman (2002). "Patriotism vs. Protest. "In: The Christian Science Monitor, 31. Juli (http://www.csmonitor.com/2002/0731/p03s01-ussc.htm).

Jim DeRogatis (2002). »Needed: A Great 9/11 Anthem. «In: Chicago Sun-Times, 6. August (http://www.suntimes.com/output/derogatis/cst-ftr-dero06.html; http://www.jimdero.com/News2001/Aug6Anthems.htm).

Stanley Kurtz (2002). »Those 9/11 Songs: Are you with Springsteen or Keith?« In: NRO nationalreviewonline, 27. August (http://www.nationalreview.com/kurtz/ kurtz082702.asp).

David Cantwell (2002). "God Squad: Some Musicians Declare Themselves Chosen Ones, while Others Maintain God's not Taking Sides. «In: The Pitch, 29. August (http://www.pitch.com/issues/2002-08-29/interview.html).

Jim Slotek (2002). "9.11.01: Day that will Live in Irony.« In: Toronto Sun / Jam! SHOW-BIZ, 8. September (http://www.canoe.ca/JamColumnSlotek/sep8_sloteksun.html).

Dave Gil de Rubio (2002). "Tribute to Heroes: A Look at the Music Inspired by 9-11.« In: Pop + Politics, 10. September (http://www.popandpolitics.com/ articles_detail.cfm?articlelD=1127).

Lorraine Ali (2002). »The Arts after 9-11: Music. In: Newsweek Web, 12. September (http://www.msnbc.com/news/800378.asp?cp1=1).

Peter Bothum (2002). »9/11: Tempting Target for Songwriters. «In: York Daily Record, 13. September (http://ydr.com/story/living/1512; Stand v. 2.9.2003).

Eric Nuzum (2002). "Crash into Me, Baby: America's Implicit Music Censorship in the Wake of September 11th. "Paper presented at the Second World Conference on Music and Censorship, Copenhagen, 29. September (http://ericnuzum.com/ banned/articles/paper_wcmc.html) (2004 erschienen als "Crash into Me, Baby: America's Implicit Music Censorship since 11 September.« In: Shoot the Singer! Music Censorship Today. Hg. v. Marie Korpe. London: Zed Books Ltd.).

George Sanchez (2002). "Popular Music after 9/11: Putting Politics into Verse. "In: FrictionMagazine, 16. Oktober (http://www.frictionmagazine.com/song/news/ 911 music.asp). 
Walkin' T:-)M (2002). »World's Out Of Tune (3): The >War on Terrorism< in Song and Poetry.« In: Folk World, the online music magazine, Nr. 24 (Dezember) (http://www.folkworld.de/24/e/ground0.html).

Jim Beal (2002). »9-11 Attacks Inspire Songwriters. "In: San Antonio Express-News, ohne Dat. (http://www.capecodonline.com/capesounds/songwrite16.htm).

Randy Lewis (2003). »The Grammys: Reverberation of 9/11 Heard in Voters' Nods." In: Los Angeles Times, 8. Januar (http://www.calendarlive.com/music/ grammy/cl-et-lewis8jan08.story; Stand v. 2.9.2003).

Edna Gundersen (2003). »Artists Mount a Chorus Against War.«In: USA TODAY, 27. Februar (http://www.usatoday.com/life/music/news/2003-02-27-community_x. htm).

Craig Havighurst (2003). "Iraq War Issue Splits Music Industry Here.« In: Nashville Tennessean Online, 4. März (http://www.tennessean.com/local/archives/03/ 03/29688900.shtml?Element_ID=29688900).

Dan Deluca (2003). »Musicians Chiming in, Pro and Con, on Iraq. «In: The Philadelphia Inquirer, 11. März (http://www.kansas.com/mld/kansas/entertainment/ movies/5358903.htm).

Jon Pareles (2003). »Protest Singers Call up their Reserves. «In: The New York Times, 12. März (http://www.iht.com/articles/89504.html).

Howard Cohen (2003). »Number One with a Bullet?« In: The Miami Herald, 24. März (http://www.miami.com/mld/miamiherald/5465355.htm).

Corey Moss (2003). »Lenny Kravitz, R.E.M. Record Anti-War Songs. "In: MTV News, 25. März (http://www.mtv.com/news/articles/1470746/03252003/kravitz lenny.jhtml).

Brian Orloff (2003). »Thurston Moore, R.E.M. Protest War with Music.«In: neumu, 25. März (http://www.neumu.net/datastream/2003/2003-00040/2003-00040_ datastream.shtml).

Sean Hackbarth (2003). »Pop Music in a Time of War.«In: Enter Stage Right, 31. März (http://www.enterstageright.com/archive/articles/0403/0403popmusic. htm).

Jim DeRogatis (2003). "Pop Music and the Battle Joined. In: Chicago Sun-Times, 2. April (www.suntimes.com/output/ derogatis/cst-ftr-dero02intro.html; http: //www.jimdero.com/News2003/WarSongsApr2.htm).

Anon. (2003). "What >Music Stars « are Saying about the War on Iraq«. In: Radio One, o.D. [April] (http://www.radiooneglobal.com/war/main.htm).

\section{HipHop}

Jeff Chang (2001). »Hip-Hop Must Call for Peace.«In: Counterpunch, 17. September (http://www.counterpunch.org/chang.html).

Davey D (2001). »Boots Speaks out about 9-11.« In: Davey D's Hip Hop Corner, 20. September (http://www.daveyd.com/bootsonthewarpolitics.html).

Jeff Chang: »Clear Channel Fires Davey D. «In: CounterPunch, 3. Oktober (http:// www.counterpunch.org/chang2.html).

Davey D (2001). "Silence Is Golden: Hip Hop Watch Your Back. «In: Hip Hop News FNV Newsletter, o.D. [Oktober] (http://www.daveyd.com/silenceisgolden. html).

Davey D (2001). »Paris Responds to the War on Terrorism. « In: Davey D's Hip Hop Corner, Oktober (http://www.daveyd.com/parisrespondsback.html). 
Kevin Weston (2001). " Osama's Mama - Corporate Hip-Hop Promotes U.S. War.« In: Pacific News Service, 5. November (http://www.corpwatch.org/article. php?id=763).

Dorian Lynskey (2002). »Draft Me!« In: The Guardian, 22. Januar, S. 12-13 (http://www.guardian.co.uk/arts/story/0,3604,637224,00.html).

Adam Heimlich (2002). »2002: Hiphop's Year One. Nas, Mobb Deep and Wu Face 9/11. In: New York Press 15, Nr. 4 (23.-29. Januar) (http://www.nypress.com/ 15/4/news\&columns/feature.cfm).

Jeff Chang (2002). "9/11: The Fallout.« In: Bad Subjects, Nr. 59 (Februar) (http://eserver.org/bs/59/chang.html).

Jeff Chang (2002). "Styling \& Profiling: Privacy \& the Hip Hop Generation after 9/11.« In: Davey D's Hip Hop Corner, 24. März (Presented at Media Bistro Salon, 19.3.2002) (http://www.daveyd.com/stylingprofilingchang.html).

Kevin Powell \& Charlie Braxton (2002). "Conversations with Hip Hop Journalists Kevin Powell \& Charlie Braxton on 9-11 [Parts 1-4].« In: Davey D's Hip Hop Corner, 24. März (http://www.daveyd.com/kevincharliewarpt1.html).

Brian Goedde (2002). "9/11 as Muse: Local Rappers Respond with Posturing, Poetry.« In: The Stranger 11, Nr. 31 (18.-24. April) (http://www.thestranger. com/2002-04-18/music.html).

Jeff Chang (2002). »HipHop's 9/11 Blues: Bush's War Takes Hard Edge Off Rap's Kickass Message. «In: NOW online edition, 25 April (http://www.nowtoronto. com/issues/2002-04-25/news_story.php).

Temple of Hip Hop Ministries... (2002). »The Spiritual Meaning of 9-11 (Hiphop Appreciation Week, May 13th-20th, 2002). «In: Untamedbrain (Miami), o.D. [Mai] (http://www.geocities.com/tonyv121/Spiritual911.html; Stand v. 2.9.2003).

Walidah Imarisha (2002). »Hip Hop Confronts War.«In: WarTimes, o.D. [August] (http://www.war-times.org/issues/4art9.html).

Al Giordano (2002). »Eminem's Antidote to September 11th: The Rapper >With a Plan to Ambush this Bush Administration - Conquers the Music Charts. «In: The Narco News Bulletin, 12. August (http://www.narconews.com/eminem1.html; Reaktionen: http://www. narconews.com/letters0802.html).

Nolan Strong (2002). "9-11: Hip-Hop Reflects. In: ALLHIPHOP.com, September (http://www.allhiphop.com/features/?ID=431).

Chuck > Jigsaw Creekmur (2002). "9-11 Is Not A Joke: Hip-Hop Processes Last Year's Terrorist Attacks.«In: BET.com, 9. September (http://www.bet.com/articles/ 0, ,c3gb3793-4477,00.html).

Tim Adkins (2002). »What Went Down at KRS' 9-11 Conference." In: Davey D's Hip Hop Daily News, 16. September (http://www.daveyd.com/FullArticles/ articleN1254.asp).

Davey D (2002). "We Going to War - Hip Hop Weighs in. In: Davey D's Hip Hop Daily News, 11 October (San Jose Mercury Column) (http://www.daveyd.com/ FullArticles\%5CarticleN1296.asp).

Tim Adkins (2002). »Hiphop and Sept. 11th.« In: Tha Formula.Com Records, o.D. [Oktober] (http://www.thaformula.com/temple\%20of\%20hip\%20hop.htm).

Anon. [FPA: The Foundation for a Patriotic America] (2003). "Hip Hop: The Sound of Terrorism. Ban Rap for the Sake of our Children - and America. In: Patrioticamerica.net, 13. Februar (http://www.patrioticamerica.net/fea3.html).

Rob Thomas (2003). "Hip-hop and the War.« In: The Capital Times / Wisconsin State Journal, 9. April (http://www.madison.com/captimes/features/stories/ 46595.php; http://static.highbeam.com/w/wisconsinstatejournal/april092003/ hiphopampthewar). 


\section{Country Music}

Ronnie Pugh (2001). »From >Dixie< to >God Bless The U.S.A.<: Country Music at War.« In: VH1.com, 28. September (http://www.vh1.com/thewire/content/ news/1449410.jhtml).

Brian Mansfield (2002). »Country Music, in 9/11 Time."In: USA TODAY, 6. Juni (http://www.usatoday.com/life/music/2002-09-05-sept11-country_x.htm).

Sue Keogh (2002). "September 11: Country Music's Biggest Test. "In: BBC Radio 2, September (http://www.bbc.co.uk/radio2/country/features/feat_911.shtml; Stand v. 2.9.2003).

Chet Flippo (2002). »Nashville Skyline: 9/11 Songs: Real People Doing the Right Thing in Life. " In: CMT/CMT.com, 12. September (http://www.cmt.com/news/ articles/1457412/09102002/id_0.jhtml)

Jaye Albright [2002]. »Reasons for Success - Fall 2001.« In: McVay Media Network (http://www.mcvaymedia.com/country/02articles/reasonsforsuccess.htm).

Jaye Albright [2002]. »@Music/Country: Post 9/11 Mid-Fall Overview. In: McVay Media Network (http://www.mcvaymedia.com/country/post9_11overview. htm).

William MacDougall (2003). "Country Music and the Crimes of Patriotism. The Song Remains the Same. Georgie on My Mind.«In: Counterpunch, 5. April (http:// www.counterpunch.org/macdougall04052003.html).

\section{Stimmen aus der BRD}

Tino Hanekamp (2001). "Die Musikwelt nach dem Attentat. Spenden, Statements, Schwachsinn. «In: Telepolis - Magazin der Netzkultur, 21. September (http:// www.heise.de/tp/deutsch/inhalt/musik/9617/1.html).

Diedrich Diederichsen (2001). "Das WTC hat es gegeben.« In: taz, Nr. 6567 (6. Oktober), S. 13-14 (http://www.taz.de/pt/2001/10/06/a0100.nf/text).

Tobias Lindemann (2001). »A New Morning, Changing Weather?« In: Raumzeit, Nr. 12 (Dezember) (http://www.raumzeit-online.de/122001/17.html).

Dietmar Kesten (2002). „Wohin gehen wir? Drei Songwriter zum 11. September 2001.« In: trend onlinezeitung, August (http://www.trend.infopartisan.net/ trd0902/t210902.html).

Tino Hanekamp (2002). »Pop-Protest gegen Kriegstreiberei.«In: Telepolis - Magazin der Netzkultur, 22. August (http://www.heise.de/tp/deutsch/inhalt/musik/ 13129/1.html).

Günther Jacob (2002). »'Don't Believe The Hype . Antisemitismus im US-amerikanischen HipHop. «In: Iz3w, Blätter des Informationszentrums 3. Welt, Nr. 263 (September), S. 32-35 (http://www.klick-nach-rechts.de/gegen-rechts/2002/ 09/hiphop.htm).

Martin Büsser (2002). »Let's Roll the Freedom - Pop nach dem 11.9.«In: Konkret, Nr. 9 (September), S. 41.

Klaus Walter (2002). »Alles war, wie es vorher ist. Zum 11. September spuckt die Kulturindustrie ihre Memos aus." In: Jungle World, Nr. 38 (11. September) (http://www.nadir.org/nadir/periodika/jungle_world/_2002/38/21a.htm). 
Klaus Walter (2002). »Pop goes Krisenstabslogik.« In: taz, Nr. 6850 (11. September), S. 21 (http://www.taz.de/pt/2002/09/11/a0134.nf/text).

Andrian Kreye (2002). »11. September - Der Terror und die Folgen. Sehnsucht nach Hymnen und Kindheit. " In: Süddeutsche Zeitung, 11. September (http:// users.rcn.com/akreye/9-11-Feui.html; Stand v. 2.9.2003).

Meike Schnitzler (2002). »Der Schock des Erwartbaren.«In: Welt am Sonntag, 25. September (http://www.welt.de/daten/2002/07/21/0721mm345687.htx).

Gerhard Maier (2002). »'Mit Rücksichtnahme auf Empfindlichkeiten<. Zum neuen Schmusekurs der Popkultur nach dem 11. September.« In: The News Blast, Mundwerk, o.D. (http://www.newsblast.de/mundwerk/newpop1.php).

Martin Büsser (2003). »Pop, Protest und World Wide Web. Wie man sich gegen eine doppelte Zensur wehrt.«In: NMZ 52, Nr. 3 (Februar), S. 30 (http://www.nmz. $\mathrm{de} / \mathrm{nmz} / 2003 / 03 /$ verdi.shtml).

Thomas Gross (2003). »Alter Protest in neuen Klamotten. Von Robbie Williams bis Herbert Grönemeyer sind gegen den Krieg. Doch schmal ist der Grat zwischen Engagement und Friedenskitsch. «In: Die Zeit, Nr. 10 (27. Februar), S. 37.

Udo Feist (2003). »Hengstberuhigung. Die Rückkehr des wohltemperierten Songs." In: Popvisionen. Links in die Zukunft. Hg. v. Klaus Neumann-Braun, Axel Schmidt u. Manfred Mai. Frankfurt/M.: Suhrkamp, S. 134-153.

Bänz Friedli und Philip Wegmüller (2003). „Ein bisschen Frieden. ...ein bisschen Protest, aber bitte nur leise. Die Stars der Pop-Industrie schmücken sich mit pazifistischen Accessoires, doch eine neue Friedens-Hymne fehlt. «In: FACTS 13, 27. März (www.a-vela.de/p34.html+irak+song+rap\&hl=de\&tie=UTF-8; Stand v. 2.9.2003).

Janko Röttgers (2003). »Der Kultur-Krieg. Radio- und Fersehsender streichen Antikriegs-Songs aus dem Programm, Musiker wehren sich mit MP3s. "In: Telepolis Magazin der Netzkultur, 27. März (http://www.heise.de/tp/deutsch/inhalt/ musik/14475/1.html).

Marit Hofmann (2003). "Popkameraden. Von der Popfraktion bis zu den Schlagerfreunden - alle leisten ihren humanitären Einsatz für die deutsche Außenpolitik.« In: Konkret, Nr. 4 (April), S. 51 (http://www.konkret-verlage.de/kvv/ txt.php?text=popkameraden\&jahr=2003\&mon=04).

Hannes Loh (2003). »'Kraß wie die Hamas - Wenn ein deutscher HipHopper gegen 'die Amerikaner wettert, sind antisemitische Stereotype nicht weit.« In: Konkret, Nr. 4 (April), S. 79.

Steve Austin (Freundeskreis Antifa Duisburg) (2003). »Deutsche Pop-Kultur im Friedenstaumel.« In: T-34 [Zeitung der Antifa-Duisburg], April (http://www.antifaduisburg.de/popfrieden.htm). 